\title{
Reaproveitamento do sangue em cirurgia com circulação extracorpórea: utilização de processadora por fluxo descontínuo
}

Maurício GALANTIER*, Rolf Francisco BUB*, José Luiz GHIOTTO*, Roberto B. da TRINDADE*, Fernando Soares da SILVEIRA*, Nelson HAMERSCHLAK*, Leonel SZTERLING*, Kelcen Diniz GOMES*, Jozef FÉHER*

GALANTIER, M.; BUB, R.F.; GHIOTTO, J.L.; TRINDADE, R.B.; SILVEIRA, F.S.; HAMERSCHLAK, N.; SZTERLING, L.; GOMES, K.D.; FÉHER, J. - Reaproveitamento do sangue em cirurgia com circulação extracorpórea: utilização de processadora por fluxo descontínuo. Rev. Bras. Circ. Cardiovasc., 2 (1): 70 - 74 , 1987.

RESUMO: Os autores, seguindo uma tendência de inúmeros Serviços, têm procurado técnicas visando reduzir, e em alguns casos eliminar, a utilização de sangue e derivados, em pacientes submetidos a cirurgia cardíaca com circulação extracorpórea. Tal fato objetiva reduzir os riscos de morbidade e de transmissāo de doenças (principalmente hepatite e AIDS), relacionados à transfusão sangüinea. Desta forma, analisam 50 pacientes submetidos a cirurgia com circulação extracorpórea, nas quais foram utilizadas técnicas de autotransfusão pré ou transoperatória, hemodiluição total durante perfusão, reaproveitamento do sangue aspirado no campo operatório e reaproveitamento do sangue residual do oxigenador, processando-os em aparelhos de fluxo descontinuo. Com estes métodos, houve uma significativa redução no volume transfundido ( $324 \mathrm{ml}$ em média no transoperatório e $272 \mathrm{ml}$ no pós-operatório), sendo que $34 \%$ dos pacientes não receberam sangue no transoperatório; $36 \%$ não o utilizaram no pós-operatório e $20 \%$ não o fizeram em todo o período hospitalar. Também não houve anemia significativa (hematócrito no pós-operatório imediato de $38,4 \%$ e, no 5 : dia de pós-operatório, de $35,9 \%$, o que diminui os riscos a ela relaconados, como astenia, sonolência, inatividade, secreção pulmonar, fenômenos trombo-embólicos, etc., principalmente em pacientes idosos, sem influência no custo total de uma cirurgia com circulação extracorpórea.

DESCRITORES: autotransfusâo; sangue, reaproveitamento; circulação extracorpórea, reaproveitamento de sangue.

\section{INTRODUÇÃO}

Desde os primórdios da cirurgia cardíaca, tem existido uma preocupação com a redução das necessidades da transfusão de sangue e derivados nos períodos trans e pós-operatórios ${ }^{4}$. Tal fato relaciona-se à incidência de complicações correlatas às transfusões, como reações febrís, isoimuni- zações, hepatite, malária, doença de Chagas e outras doenças infecciosas, tendo-se acentuado com o surgimento de casos de síndrome de imuno deficiência adquirida (AIDS) por transmissão transfusional ${ }^{1,7.8}$.

Várias técnicas têm sido preconizadas, no sentido de reduzir o volume transfundido, como utiliza-

Trabalho realizado no Hospital Israelita Albert Einstein, São Paulo, SP, Brasil.

- Do Hospital Israelita Albert Einstein.

Entregue para publicação em 22 de abril de 1987.

Endereço para separatas: Mauricio Galantier, Av. Albert Einstein, n: 627. 05652 Sâo Paulo, SP, Brasil. 
ção de perfusão não sangüínea (hemodiluição to(al) ${ }^{4}$, autotransfusão pré e/ou transoperatória ${ }^{12}$, reaproveitamento do sangue aspirado no campo operatório e utilização do sangue drenado no pós-operatório ${ }^{5,6} *$ *10-13. Em algumas dessas técnicas, o sangue, antes de ser reinfundido, pode ser processado em lavadoras automáticas ou aparelhos de fluxo descontínuo Dideco, Haemonetics, etc.), de tal forma a reaproveitar, fundamentalmente, hemácias, eliminando-se estroma celular, hemoglobina plasmática livre, enzimas séricas e celulares ativadas, agregados de proteínas, produtos de degradação de fibrina, anticoagulantes, etc. ${ }^{1}$.

Observa-se que, com essas técnicas, os pacientes podem evoluir com anemia significativa, chegando a níveis de hematócrito ao redor de $22-23 \%{ }^{7}$. Esta anemia, embora normovolêmica, pode aumentar a morbidade, principalmente em pacientes idosos.

Os Serviços de Cirurgia Cardiovascular e Hemoterapia do Hospital Israelita Albert Eistein, em São Paulo, têm utilizado técnica de autotransfusão e recuperação de sangue com auxílio de processadoras, com o intuito básico de reduzir a necessidade de transfusões homólogas, sem determinar níveis significativos de anemia. A análise dos resultados obtidos em 50 pacientes constitui o objetivo deste estudo.

\section{MATERIAL E MÉTODO}

No período de abril de 1986 a janeiro de 1987, foram analisados 50 pacientes operados no Hospital Israelita Albert Einstein.

Desses pacientes, 45 (90\%) eram do sexo masculino. A idade média foi de 62,3 anos, variando de 36 a 72 anos; 46 foram submetidos a cirurgia de revascularização do miocárdio e 4 a cirurgia valvar (aórtica e mitral). Não foram excluídos pacientes em uso de antiagregantes plaquetários no pré-operatório ( $31 \%$ dos mesmos).

A duração média da circulação extracorpórea foi de 68,2 minutos $( \pm 23,6$ ).

A anticoagulação, durante a circulação extracorpórea, foi realizada com dose de heparina de $5,0 \mathrm{mg} / \mathrm{kg}$ de peso, efetuando-se controle laboratorial para confirmar níveis adequados. A dose de protamina deve ser cuidadosamente estabelecida, uma vez que o processamento do sangue elimina heparina do sistema. Desta forma, temos feito a correção em $1 \mathrm{mg}$ de protamina para $1 \mathrm{mg}$ de hepa- rina total utilizada, sendo injetados $60 \%$ deste valor diretamente na aorta e os restantes $40 \%$, injetados de forma endovenosa, controlando-se, laboratorialmente, a neutralização. A quantidade administrada via endovenosa pode ser parcial, ou total, uma vez que a utilização excessiva de protamina pode ter efeitos secundários, alterando a coagulação.

Foram utilizadas as seguintes técnicas, no sentido de reduzir a necessidade transfusional:

1) Coleta de sangue pré-operatória: alguns dias antes da cirurgia retiraram-se $500 \mathrm{ml}$ de sangue, os quais foram reinfundidos após a circulação extracorpórea. Esta técnica foi pouco utilizada, uma vez que a maioria dos pacientes foi operada 1 a 2 dias após o diagnóstico e em outras situações havia instabilidade hemodinâmica que não permitia tal procedimento.

2) Autotransfusão transoperatória: logo após a abertura do pericárdio, um catéter é colocado no átrio direito, sendo coletados $600 \mathrm{ml}$ de sangue, para reinfusão no período pós-extracorpórea.

3) Hemodiluição total: utilizando-se Ringer lactato e albumina como perfusato.

4) Reaproveitamento do sangue restante no oxigenador: o sangue é parcialmente reinfundido de forma direta (de acordo com as condições hemodinâmicas), ou é processado em sistema fechado, através de um aparelho de fluxo descontínuo (Dideco-Progress), eliminando-se plasma hemolisado, restos celulares, excesso de heparina e reinfundido-se concentrado de hemácias com hematócrito em torno de $70 \%$.

5) Reaproveitamento do sangue aspirado: consiste na aspiração de sangue do campo operatório, antes e após circulação extracorpórea, para o reservatório de cardiotomia, anticoagulando-se com solução heparinizada (25.000 u de heparina/ litro de solução fisiológica), gota a gota, junto da ponta do aspirador do campo cirúrgico. Este sangue é, subseqüentemente processado em equipamento de fluxo descontínuo, lavando-se e reinfundindo-se os glóbulos vermelhos. As transfusões de hemácias, no período transoperatório, basearamse em níveis de hematócrito abaixo de $29 \%$, dependendo da idade e/ou das condições clínicas do paciente.

\section{RESULTADOS}

1) Coleta de sangue pré-operatória e transoperatória: no período pré-operatório, em apenas 
GALANTIER, M.; BUB, R. F.; GHIOTTO, J. L.; TRINDADE, R. B.; SILVEIRA, F. S.; HAMERSCHLAK, N.; SZTERLING, L.; GOMES, K. D.; FÉHER, J. - Reaproveitamento do sangue em circulação extracorpórea: utilização de processadora por fluxo descontínuo. Rev. Bras. Cir. Cardiovasc., 2(1):70-74, 1987

$10 \%$ dos pacientes, foram retirados $500 \mathrm{ml}$ de sangue, 48 a 72 horas antes da cirurgia. Durante o ato cirúrgico, foram colhidos $500 \mathrm{ml}$ de sangue, em 36 pacientes $(72 \%)$. Em ambas as situações, esse volume foi reinfudido após o término da circulação extracorpórea (quando se iniciou a infusão de protamina).

2) Reaproveitamento do sangue aspirado: em 18 pacientes esta técnica foi utilizada, obtendo-se um volume de 200 a $400 \mathrm{ml}$, que foi processado, eliminando-se plasma hemolisado, resíduos teciduais, etc., permitindo reinfusão de 120 a $210 \mathrm{ml}$ de concentrado globular.

3) Reaproveitamento do sangue do oxigenador: em todos os pacientes esta técnica foi realizada. O volume processado nos aparelhos de fluxo descontínuo foi em média $1,194 \mathrm{ml}$, obtendo-se concentrado globular num volume médio de $630 \mathrm{ml}$.

4) Transfusão homóloga: durante o período transoperatório, os pacientes receberam, em média, $324 \mathrm{ml}$ de sangue, sendo que 17 (34\%) não receberam sangue nesta fase. No pós-operatório, o volume médio de sangue, ou concentrado globular, utilizado foi de $272 \mathrm{ml}$ e $18(36 \%)$ pacientes não receberam sangue, ou derivados, nesta fase. No total, $20 \%$ dos pacientes não receberam sangue em toda a permanência hospitalar e o volume médio transfundido foi de $596 \mathrm{ml}$ (Tabela 1 ).

TABELA 1

VOLUME MEDIO DE SANGUE TRANSFUNDIDOE PORCENTAGEM DE PACIENTES QUE NÃO RECEBERAM TRANSFUSĀO

\begin{tabular}{llll}
\hline $\begin{array}{l}\text { SANGUE } \\
\text { TRANSFUNDIDO }\end{array}$ & $\begin{array}{l}\text { TRANS. } \\
\text { OPERATORIO }\end{array}$ & $\begin{array}{l}\text { POS. } \\
\text { OPERATÓRIO }\end{array}$ & TOTAL \\
Volume médio $(\mathrm{ml})$ & 324 & 272 & 596 \\
Sem transiusáo & 1750 & 1850 & 10550 \\
& $34 \%$ & $36 \%$ & $20 \%$
\end{tabular}

$12 \%$ dos pacientes $(6)$ receberam concentrado de plaquetas, pelo fato de terem utilizado antiagregantes plaquetários como medicação prévia, e terem apresentado sangramento maior que o habitual.

5) Hematócrito: o comportamento do hematócrito pode ser avaliado no Gráfico 1. Os valores pré-operatórios $(43,06 \%$ em média) reduziram-se para $27,6 \%$, com a hemodiluição durante a circula- ção extracorpórea, passando os valores a $41,9 \%$ após infusão das hemácias processadas, mantendo-se em níveis de $38,4 \%$ no pós-operatório imediato e $35,9 \%$ no 5 . dia de pós-operatório.
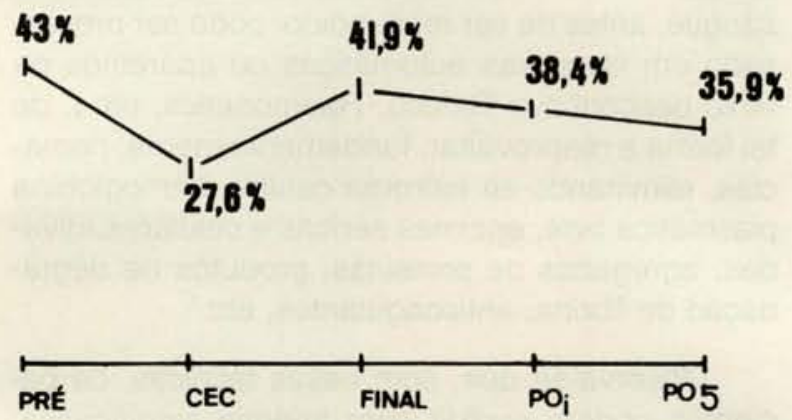

Graf. 1: Comportamento dos niveis de hematócrito do pré-operatório até o 5: dia de pós-operatório.

6) Provas de coagulação: Os testes de coagulação realizados (tempo de protrombina, tempo de tromboplastina parcial, tempo de trombina, dosagem de fibrinogênio e contagem de plaquetas) podem ser observados na Tabela 2. A heparinização, na dosagem proposta, foi considerada eficiente, bem como a neutralização, sendo utilizado em média $1 \mathrm{mg}$ de protamina por cada $\mathrm{mg}$ de heparina total injetada. O comportamento das plaquetas e fibrinogênio (queda de $50 \%$ e $31 \%$ respectivamente) está dentro dos padrões habituais ${ }^{\star 1}$.

TABELA 2

CONTROLE LABORATORIAL DOS TESTES DE COAGULAÇÃO

\begin{tabular}{lllll}
\hline & ANTES CEC & DURANTE CEC & FINAL CIRURG. & $\begin{array}{l}\text { POS-OPER. } \\
\text { IMEDIATO }\end{array}$ \\
& & & & \\
Tempo protrombina $(\mathrm{seg}) 85,5 \%(15,4)$ & $>300$ & $50 \%(20,5)$ & $61,1 \%(18,5)$ \\
Tempo tromboplastina & 51,2 & $>300$ & 57,6 & 51,9 \\
Tempo trombina (seg) & 14,6 & $>300$ & & 14,9 \\
Plaquetas mm $^{3}$ & 256.200 & 158.300 & 128.400 & 166.300 \\
Fibrinogênio (mg dil) & 283 & 198 & 198 & 237 \\
\hline
\end{tabular}

7) Morbidade e mortalidade: não houve, neste grupo de pacientes, e não foram registradas maiores complicações, principalmente no que se refere a sintomas de anemia (astenia, sonolência, etc.). A alta hospitalar foi, em média, no 8․ dia de pós-operatório.

\section{DISCUSSÃO}

Niveis significativos de complicações relacionadas à transfusão de sangue e derivados, têm

\footnotetext{
*' GALANTIER, M.; BUB, R. F.; GHIOTTO, J. L.; TRINDADE, R. B.; KORKES, H.; KNOBEL, E.; HERYNKOPK, F.; ROSENFELD, L. G. M.; SZTERLING, G. - Controle de coagulação em pacientes submetidos a circulação extracorpórea. Apresentado ao 34 : Congresso Brasileiro de Cardiologia. Belo Horizonte, 1978
} 
sido descritos. COLLINS et alii ${ }^{3}$ relatam que, para cada 1.000 unidades de sangue transfundidas, ocorre 1 óbito relacionado ao procedimento; para cada 100 unidades ocorre 1 caso de hepatite diagnosticada clínica e laboratorialmente e que, para 10 unidades, ocorre 1 caso em que existem alterações da função hepática. Estudos relativos à síndrome de imuno-deficiência adquirida (AIDS) mostram que, em 1 a $2 \%$ dos casos, a transmissão ocorreu devido à transfusão ${ }^{8} \mathrm{e}$ que, em doadores nos quais se realizaram testes sorológicos de triagem, a incidência de casos positivos foi de $0,24 \%$ em $1985^{\circ}$, e aproximadamente o dobro, em 1986.

Desta forma, todas as medidas que visam à ređuçāo do volume de sangue e derivados necessários, durante cirurgias cardiacas, devem ser utilizadas. Assim, muitos Serviços têm reduzido significativamente a necessidade transfusional ${ }^{3} \mathrm{e}$ alguns chegam a valores mínimos, como COSGROVE et alii ${ }^{6}$, que têm utilizado sangue em apenas $10 \%$ dos pacientes submetidos a circulação extracorpórea, aceitando o quadro de anemia normovolêmica, com valores de hematócrito caindo a níveis de 20 a $22 \%$ por paciente operado, em comparação com uma necessidade, em anos anteriores, de 1500 a $1800 \mathrm{ml}$. Por outro lado, na presente série, não houve queda tão acentuada do hematócrito (38,4 a 35,9\%, no período pós-operatório), determinando uma evolução bastante satisfatória, uma vez que niveis abaixo de 27 e $28 \%$, principalmente em pacientes acima de 65 anos, propiciam um quadro de sonolência, adinamia, inatividade, etc., levando a um risco maior de complicações respiratórias, vasculares periféricas $\mathrm{e}$, conseqüentemente, embolias pulmonares. Ainda podemos acrescentar que necessidades transfusionais de 1 a 2 unidades possibilitam uma seleção mais rigorosa de doadores, em geral utilizando familiares, ou do circulo de amigos do operado, com morbidade bastante reduzida*1

Acreditamos que, entre as técnicas utilizadas para este fim, o processamento de sangue residual no oxigenador seja a mais eficaz, no sentido de permitir maior concentração de hemácias, evitando niveis significativos de anemia. A infusão simples do perfusato, advogada por alguns autores ${ }^{12}$, por aproveitar fração plasmática e, nela, fatores de coagulação, nem sempre pode ser total, devido às condiçōes hemodinâmicas (hipervolemia). Nestas situações, o sangue poderia ser coletado em recipientes apropriados e transfundido posteriormente, o que, no entanto, determinaria maior risco de contaminação. Nos procedimentos por nós utilizados, emprega-se um circuito fechado entre oxigenador, reservatório de cardiotomia, processadora e paciente.

Não temos realizado o aproveitamento do sangue drenado pelo mediastino, como preconizam COSGROVE et alii, 5, 6 , GALANTIER et alii ${ }^{* 2}$, HAMERSCHLAK et alii ${ }^{7 .}{ }^{8}$, MORAN et alii ${ }^{10}$ e TECTOR et alii ${ }^{11}$. No entanto, esta técnica deve ser considerada, principalmente nos pacientes nos quais o sangramento seja importante durante o fechamento do tórax, ou nas primeiras horas pós-operatórias. Nestes casos, deve-se colocar um reservatório de cardiotomia entre o dreno mediastinal e o sistema de aspiração, sendo o sangue filtrado no reservatório e, a seguir, reinfundido ao paciente, diretamente, ou após processamento, usando-se o mesmo kit da processadora utilizada durante a cirurgia.

\footnotetext{
*' GALANTIER, M.; BUB, R. F.; GHIOTTO, J. L.; TRINDADE, R. B.; KORKES, H.; KNOBEL, E.; HERYNKOPK, F.; ROSENFELD, L. G. M.; SZTERLING. G. - Controle de coagulação em pacientes submetidos a circulação extracorpórea. Apresentado ao 34: Congresso Brasileiro de Cardiologia. Belo Horizonte, 1978.

*2 GALANTIER, M.; HAMERSCHLAK, N.; FEHER, J.; SZTERLING, L.; SZTERLING, G.; ROSENBLIT, J.; GUeRRA, C. C. C.; KORKES, H. - Doaçăo comunitária de sangue em pacientes submetidos a cirurgias com circulaçāo extracorpórea. Apresentado ao 6 : Congresso da Fundação Panamericana Pró-Doaçăo de Sangue, Brasilia, 1980.
} 
GALANTIER, M.; BUB, R. F.; GHIOTTO, J. L.; TRINDADE, R. B.: SILVEIRA, F. S.: HAMERSCHLAK, N.: SZTERLING, L.: GOMES, K. D.: FÉHER, J. - Reaproveitamento do sangue em circulação extracorpórea: utilização de processadora por fluxo descontinuo. Rev. Bras. Cir. Cardiovasc., 2(1):70-74, 1987

RBCCV

GALANTIER, M.; BUB, R. F.; GHIOTTO, J. L.; TRINDADE, R. B.: SILVEIRA, F, S.; HAMERSCHLAK, N.: SZTERLING, L.; GOMES, K. D.; FEEHER, H. - Re-utilization of blood in surgery with extracorporeal circulation: utilization of discontinous flow procedure. Rev. Bras. Cir. Cardiovasc., 2(1):70-74, 1987

ABSTRACT: The authors, following the trends of several Services, have been searching for techniques aiming at reducing, and in some cases eliminating the utilization of blood and derivatives in patients submitted to heart surgery and extracorporeal circulation. This has as objetive to reduce the morbidity as well as avoiding diseases transmission (mainly hepatitis and AIDS) related to blood transfusion. Fifty patients submitted to surgery with extracorporeal circulation were analyzed. Pre or trans-operative auto-transfusion. total hemodilution during perfusion, re-utilization of suctioned blood as well as re-utilization of the oxygenative residual blood (by processing them in discontinuous flow procedure were used. With those methods, there was a significant reduction of transfused volume $(324 \mathrm{ml}$ in average at trans-operative and $272 \mathrm{ml}$ at post-operative period): $34 \%$ of patients did not receive blood during operation: $36 \%$ did not utilize post-operatively and $20 \%$ did not use it during the whole period of hospitalization. Also significant anemia was not noted (hematocrit in immediate post-operative was $38.4 \%$ and at the 5 th post-operative day $35.9 \%$ ) what lessens the risk of asthenia, sleepness, inaction, lung secretion and thromboembolic phenomenon, mainly in elderly patients, without interferring in the total cost of surgery.

DESCRIPTORS: auto-transfusion, blood re-utilization; extracorporeal circulation, blood re-utilization.

\section{REFERÊNCIAS BIBLIOGRÁFICAS}

1 AUTOTRANS BT 975/P "PROCESSOR" Technical Report. Modena, Itália, DIDECO, 1985.

2 BAYER, W. L.; COENEN, W. M.; JENKINS, D. C.; ZUCKER, M. L.; - The use of blood and blood components in 1976 patients undergoing open-heart surgery. Ann. Thorac. Surg., 29(2): 117-122, 1980.

3 COLLINS, J. A. - Problems associated with the massive transfusion of stored blood. Surgery, 75(2): 274-295, 1974.

4 COOLEY, D. A.; BEALL Jr., A. C.; GRONDIN, P. - Openheart operations with disposable oxygenators, 5 per cent dextrose prime, and normothermia. Surgery, 52(1): 713-719, 1962.

5 COSGROVE, D. M.; AMIOT, D. M.; MESERKO, J. J. An improved technique for auto-transfusion of shed mediastinal blood. Ann. Thorac. Surg., 40(5): 519-522. 1985.

6 COSGROVE, D. M.; LOOP, F. D.; LYTLE, B. W.; GILL, C. C.; GOLDING, L. R.; TAYLOR, P. C.; FORSYTHE, S. B. - Determinants of utilization during myocardial revascularization. Ann. Thorac. Surg., 40(4): 380-384, 1985.
7 HAMERSCHLAK, N.; PASTERNAK, J.; KHOURY, A, B.: CAVAGNOLI, K.; ROSENFELD, L. G. M.; GUERRA, C. C. C.; TEIXEIRA, P. R. $-B_{2}$ Micro-globulina and anti-HTLV III in cases of AIDS: risk groups and blood donors. Ric. Clin. Lab., 16(1): 173-176, 1983.

8 HAMERSCHLAK, N. \& TEIXEIRA, P. R. - Sindrome de imuno-deficiência adquirida: hematologia e hemoterapia. Bol. Soc. Bras. Hematol. Hemot., 7(132): 37-38, 1985.

9 MENITOVE, J. E. \& KOLINS, J. eds. - AIDS. Arlington, American Association of Blood Banks, 1986.

10 MORAN, J. M.; BABKA, R.; SILBERMAN, S.; RICE, P. L.; PIFARRÉ, R.; SULLIVAN, H. J.; MONTOYA, A. Immediate centrifugation of oxygenator contents after cardiopulmonary bypass: role in maximum blood conservation. J. Thorac. Cardiovasc. Surg., 76(4): 510-517, 1978.

11 TECTOR, A. J.; DRESSLER, D. K.; GLASSNER-DAVIS, R. N. - A new method of autotransfusing blood drained after cardiac surgery. Ann. Thorac. Surg., 40(3): 305-307, 1985.

12 THURER, R. L. - Blood conservation in cardiac surgery: the role of intra operative autotransfusion. Amsterdam, Elsevier Noth Holland Inc. 1981. p. 163-170.

13 WINTON, T. L.; CHARRETTE, E. J. P.; SALERNO, T. A. - The cell saver during surgery: does it save? Ann. Thorac. Surg., 33(4): 379-381, 1982. 